\title{
AN ANALYSIS AND RISK ASSESSMENT OF VULNERABILITY MANAGEMENT PRACTICES FOR IOT DEVICES
}

\author{
Aisling O’Malley, Louise Gallagher, Ronan Cooney, Kieran Flanagan and Enda Fallon \\ Athlone Institute of Technology, Co. Westmeath, Ireland
}

\begin{abstract}
The Internet of Things (IoT) is no longer a buzz word. In place since 2008/2009 at a time when there was more "things or objects" connected to the Internet than people, there has been an exponential rise in connected devices, and according to a projection by the International Data Corporation (IDC), there will be 41.6 billion connected IoT devices, generating 79.4 zettabytes (ZB) of data by 2025 . These IoT devices can be found within our homes, businesses and almost every industry, like fitness and health care. With the increasing scale of IoT presenting on networks, comes the additional upsurge in cyber-attacks on these systems and an upsurge in management of cyber vulnerabilities is needed in parallel. This paper will review what kind of vulnerabilities IoT devices of all ages are susceptible too and what state of the art vulnerability management practices are available to address those threats. Based on findings, we will propose the use of a Vulnerability Risk Assessment Tool (VRAT) to determine the approximate risk an IoT device could pose, as well as issue a recommended vulnerability management action for that device based on the risk rating.
\end{abstract}

\section{KEYWORDS}

Cybersecurity, Risk Analysis, Internet of Things (IoT)

\section{INTRODUCTION}

Cisco IBSG has predicted that there will be approximately 50 billion devices connected to the Internet by 2020 (Evans, 2011). In parallel, research (Harwood, 2019) has determined that existing IoT devices have almost double the amount of vulnerabilities compared to six years ago, despite a marked increase in security awareness and knowledge.

The number one beneficial characteristic of most IoT devices is convenience. However, from the beginning of pioneer IoT "things or objects", circa 2008/2009 (IDC, 2019) there is a predicted 41.6 billion expected to be connected by 2025 (McMahon et al,. 2017) and this prevalence has caused them to rise in the ranks as a favorite attack surface for a nefarious actor. Thus with this convenience comes the possibility of cyber-attack if vulnerabilities are not managed correctly, or at all. Despite multiple state of the art techniques to manage IoT vulnerabilities, it is evident there are still many vendors and end users not adopting proper security defenses in order to protect their gadgets.

This work proposes to (a) review critical vulnerabilities that have been identified on IoT devices of all ages. A subset of three main categories of devices, medical, home router and fitness will be used for this data. It will (b) further appraise state of the art practices that are in place to manage and eradicate these weaknesses. Research will show that studies and information are mostly limited to current and future state IoT devices, with minimal data available on vulnerabilities within older (over five - ten years) IoT devices, and additionally scarce guidelines are available on how to manage same. To address this gap, the following questions were posed as further research for this study: (a) What vulnerabilities are older IoT devices susceptible to and what measures are in place to mitigate or prevent these and (b) What measures should be in place to mitigate or prevent vulnerabilities on older IoT devices.

To fulfil this research, published data from three top medical device manufacturers are reviewed and a solution offered in the form of VRAT. This tool will take input of considerations such as the vulnerability type, the age of the device and current security controls, and output a risk rating as well as a recommended vulnerability management practice pertinent to the rating. 


\section{LITERATURE REVIEW}

\subsection{Security Vulnerabilities in Medical, Home Router and Fitness IoT Devices}

Network connected healthcare devices are creating a world of "Healthcare Internet of Things" (HIoT) which are providing a better state of monitoring and preventive care. However, security research has revealed that HIoT devices could pose an unseen danger.

A vulnerability disclosed by Johnson \& Johnson in one of their insulin pumps (Beardsley, 2016) has been shown to potentially over prescribe insulin to a patient if exploited. This weakness, discovered in the "OneTouch Ping", a device used by diabetic patients to administer insulin, is believed to be one of the first manufacturer reported warnings of a cyber security vulnerability. It was found that the pump system uses clear text communication rather than encrypted, and this lack of encryption could allow a remote attacker to spoof the meter and initialize unauthorized injections of insulin (Beavers et al., 2019).

"Hacking NHS Pacemakers" (Kirk, 2012) research proposed 3 different types of attacks that could occur on NHS standard units - signal jamming, code injection and relay attack. The results from this particular experiment found that the device tested was not vulnerable to basic attacks, however legacy devices were not factored into this investigation. The findings of renowned medical hacker "Barnaby Jack" contradicts this report. During a Breakpoint Security Conference in Melbourne, Australia, Jack demonstrated how he could remotely cause a pacemaker to deliver an 830 volt shock (Zheng et al., 2017).

In the medical industry, data protection, especially patient Personal Identifiable Information (PII) is paramount. One of the biggest ransomware attacks that was observed worldwide took hold in May 2017 and infected multiple medical facilities across the British National Health Service (Mirani et al., 2019). The Wannacry cyberattack exploited a vulnerability in Windows operating systems causing the malware to encrypt all files in the computer and hold it for a bitcoin ransom, in exchange for file access. It is not known to what extent PII was obtained and lost after this incident, but due to the scale of the attack it is believed to be huge.

The Internet Census of 2012, a research "project" that built a port scanner botnet from 420k IoT devices on the internet with weak or no credentials, found that the vast majority of those devices were consumer routers and that the $420 \mathrm{k}$ figure only accounted for approximately $25 \%$ of unprotected devices found (McMahon et al,. 2017). Whilst no recent report as extensive as this could be found, further evidence from the last three years exists to support their findings, such as that from the SOHOpelessly Broken project. This was completed over two phases, the first in 2013 and the second in 2019, with an objective of remotely obtaining root-privileged access on routers without any authentication (Level 3 Threat Research Labs, 2016). It was determined that security controls put in place by vendors of home routers were insufficient when it came to cybersecurity attack with the team acquiring root access to 12 out of the 13 devices tested.

Level 3 Threat Research Labs (now CenturyLink) identified in 2016 that the most recent distributed denial of service (DDoS) attacks recorded originated from three different types of devices $-4 \%$ of which were home routers (Angrishi, 2017). The severity of this type of attack lie in the fact that the botnets that cause DDoS can remain hidden on the device without causing any performance issues (Cyr et al., 2014). Some well-known botnet attacks that have had serious impact on routers are Carna, Chuck Noris and Mirai which Angrishi (2017) describes as having "inspired a renaissance in IoT malwares" due to the predominant large scale DDoS attacks that followed in its aftermath.

Like routers, research has been conducted into the security of IoT fitness devices. A project analyzing the security of fitness wearable devices, conducted by a group of MIT students (Lofty et al., 2016), focused on establishing the privacy and security concerns of the device, Bluetooth traffic analysis and a security posture of the Fitbit application for android users. The research demonstrated that the exposure of Bluetooth Low Energy (BTLE) credentials throughout device pairing could be utilized for man-in-the-middle (MITM) attacks as they were transmitted via plaintext. During Fitbit pairing, device information on additional Fitbits in range could be identified and JavaScript's sent to the phone could be used to generate this type of attack.

A similar research study conducted by students from the University of Nebraska highlighted that fitness devices are used to record many important security details about the consumer (Rieck, 2016). The uncertainty around what happens to the data and how it is exchanged resulted in the questioning of how safe wearable devices really are. Three types of Bluetooth pairing techniques were evaluated to determine if there were any security concerns. Upon analysis of these techniques, the groups identified that the poor security parameters in place could result in significant data exfiltration and MITM attack. Additional research by (Lofty et al., 
2016) investigates how fitness devices unnecessarily obtain information on their users without the ability for customers to obtain this data.

Alongside data concerns with fitness devices, "Attacks on Fitness trackers" (Classen et al., 2018) focused on the concept around firmware attacks. The initial findings of this report indicated that firmware updates are essential to the security of fitness devices. A subsequent report discussed an innovative firmware attack that allows malicious code to be injected. The firmware vulnerability targets the data at rest due to poor hardware configuration within the device. If left unpatched, this could result in an attacker intercepting the connection and detonating injectable, malicious code. Anatomy of a Vulnerable Fitness Tracking System (Nagy et al., 2019), which also highlighted security concerns around the lack of firmware updates on Fitbit devices, focused on encryption standards. They advocated for tighter controls which would allow for the vendor to certify that the BLE commands issued by their server are encrypted securely, in order to reduce the possibility of tampering.

\subsection{State of the Art Practices in Place to Manage and Eradicate Weaknesses}

Ensuring the security hardening of any IoT device is considered an imperative task, even more so if it is a centralized device like a home router. A hardware solution in the form of a Raspberry Pi has been determined to be a good choice for offering protection to other IoT devices on the network and eliminating external threat (Kumar et al., 2017). After adding additional security mechanisms to the device, passing packets are analyzed to detect malicious behavior patterns and an action, like blacklisting an IP, occurs to prevent the attack. For brute force password attacks, the IOC could be at least 5 attempts simultaneously by the same IP address in less than one minute, and at least 5 different usernames attempted with at least 5 different passwords attempts - the specifics would be fine-tuned to increase the integrity of the alert (Kumar et al., 2017).

An efficient DDoS prevention strategy has been outlined by (Fitbit, 2019) as consisting of three phases: DDoS detection, attack identification and DDoS defense. A Learning Automata System Model is based within these phases and an algorithm used to output an action in response to an event. Additionally, periodically monitoring of internet firewall $\operatorname{logs}$ for anomalies or suspicious traffic on ports 2323 and 23 can alert to direct denial of service attacks (Cyr et al., 2014).

To reduce concern amongst consumers of Fitbit devices, best security practices involve applying strong authentication measures to their devices and securing and locking down information on their personal accounts (Franklin, 2019). Privacy policy and how data is stored, used and protected is also available for perusal. The implementation of multifactor authentication can ensure data and devices are secure and PII and PCI data is protected (Sizemore et al., 2016). Users can disable location settings so that the devices geographical location is not being stored or tracked by Fitbit services (Marks, 2019). BTLE credentials are also considered, secure encryption by using SSL/TLS is the top recommendation.

Fitness devices are specifically designed to operate on low levels of Bluetooth energy. However, "low energy equals low security" (Hyppönen et al., 2007). Bluetooth 4.0 is predominately used for the transmission of data within fitness devices as it is designed to use low levels of energy, however, this increases the likelihood of data compromise. A simple and effective measure to ensure data is protected is by disabling Bluetooth connection and data syncing until a user is logged on to a safe and trusted network. Implementing the use of a VPN will ensure data is being transmitted securely and encrypted over an unfamiliar network (ICS-CERT Advisories, 2016). "Nĩno" (Vlajic et al., 2018) a research project that focused on MITM attacks via Bluetooth pairing has concluded that MITM are impossible to prevent while connected to a wireless network. However, it advises users can educate themselves on the awareness of cyber security threats and be mindful of where they share sensitive information.

Applying firmware updates on a fitness device generally doesn't appear too high on an end user's "to-do" list, but any device that is storing PII, PCI and PHI data should be fully secure and protected to prevent data exfiltration or other forms of cyber-attack. Devices can be kept securely up to date by verifying the latest firmware version and updating if required. Additionally, the vendor of the devices should constantly work on updating the firmware to the latest security standards.

In contrast, as observed in "Hacking NHS Pacemakers: A Feasibility Study" (Kirk, 2012), vulnerability management practices can sometimes lead to further vulnerabilities and exploits. As medical devices like pacemakers are re-programmable to ensure software can be patched and updated, this opens a possibility of code injection attacks, consequently allowing a threat actor to design malicious code to effect the device. These are further considerations that need to be top of mind for any healthcare vendor who should be ensuring that all vulnerability practices are state of the art. 
A major challenge for healthcare services observed in the papers reviewed is the vulnerability management of HIoT within the life cycle of a product. As newer products are released to the market, manufacturers can lose focus on the support of legacy devices. This was evident in the mitigation process of the vulnerabilities of "OneTouch Ping" insulin pumps (Beardsley, 2016). The manufacturer did not plan to release a firmware update to address the vulnerabilities, instead communicated a set of controls to "reduce the risk associated with the vulnerabilities" (Burhan et al., 2018) rather than try to prevent the occurrence of then, as would be good practice.

One of the biggest wake up calls for vulnerability management policy makers that saw a huge change in practices, was the Wannacry outbreak in 2017 (Mirani et al., 2019). The scale of the attack due to one patch not been deployed, was catastrophic, but drove home the urgency for patch implementation when advertised as critical by the vendor. Highlighting the dangers of enabling SMBv1, or opening port 445 and NetBIOS served as an advisory to close these by default for many organizations. In the latest version of Windows 10 and Windows server 2016 SMBv1 is not installed as standard, a dedication to hard lessons learned.

The SOHOpelessly Broken project provides insightful examination valuable to this paper. Because their work comprised of research from the earlier years of IoT devices and state of the art, they were readily able to identify any improvements or lack thereof. They noted that popular security parameters to protect against cross site request forgery or browser security headers were not commonplace, but would have protected against their exploit if in existence. Perhaps most of note from this research was that the attacks took place on fully updated devices, illustrating that the issues found lay in poor security configuration, not specifically because of lack of update as one would assume.

The prevalence of IoT devices has caused them to rise in the ranks as an attackers' favorite means to deploy a DDoS attack. IoT search engines, like Shodan, further increases their attraction by quickly identifying new vulnerable devices and allowing attacks to occur that are even more difficult to prevent or predict (Kantaria, 2018). Despite multiple state of the art techniques to manage this vulnerability, it is evident there are still many vendors and end users not adopting proper security defenses in order to protect their device.

Whilst research into fitness devices evaluated security concerns believed to be of great trepidation for end users, the conclusion was that the Fitbit device provided a reasonable level of privacy for user data, that there was a reduced risk of attack on the user's data and that Fitbit appears to have effective security measures in place that would prove difficult for their user's data to be at risk. The only remaining concern was the preference for users to easily access all the data that is recorded by the device (Lofty et al., 2016). However, of significant concern is how the data in transmission could be easily obtained by a potential attacker. The research shows that the Bluetooth fortifications currently in place don't guarantee protection for the consumers data (Rieck, 2016) unless vendors of wearable fitness devices ensure these Bluetooth and data exchange mechanisms are of a high security standard with the ability to apply encryption to the data.

With regards to the security of firmware updates on a fitness device two different variations of attack has been determined as possible, those that focus on the interference between the Bluetooth and Wi-Fi channels with an objective of manipulating the data once it is produced by the device and a subsequent "mule" attack which is a manipulation of the local environment which falsely records the steps and activities of the device (Classen et al., 2018). Additional analysis completed on Withings Activité confirmed that it was possible to inject malicious code on the device due to a restriction with the hardware configuration which resulted in a lack of privilege separation.

Whilst many reports and research exists as to how IoT devices should be vulnerability managed, and what can happen if they are not, this paper was approached with the scope of determining what vulnerabilities and security practices exist on IoT devices of all ages, both legacy and state of the art. However, we have been unable to find meaningful research and results in the pioneer area, i.e. IoT devices from its approximate age of conception, 2009, or those over five - ten years old. This gap begs the question, what are the vulnerability management practices in place for legacy IoT devices or is the focus solely on the future of IoT without a backward glance at what has gone before and that still remains in a connected state?

\section{METHODOLOGY}

In order to address research for part A, "What vulnerabilities are older IoT devices susceptible to, and what measures are in place to mitigate or prevent these?" the focus was placed on one specific area of study medical devices. The methodology used will be as follows: 
a) Using three state of the art medical device companies, determine what vulnerabilities (if any) have been found on their manufactured medical devices aged over five - ten years old.

b) Determine what vulnerability management practices (if any) are in place by the vendor to mitigate these or future vulnerabilities.

The research for this testing is conducted on top medical device vendors: Medtronic, Abbott and Boston Scientific. The initial vulnerabilities found and the frequency of vulnerabilities are listed below in Figure 1.

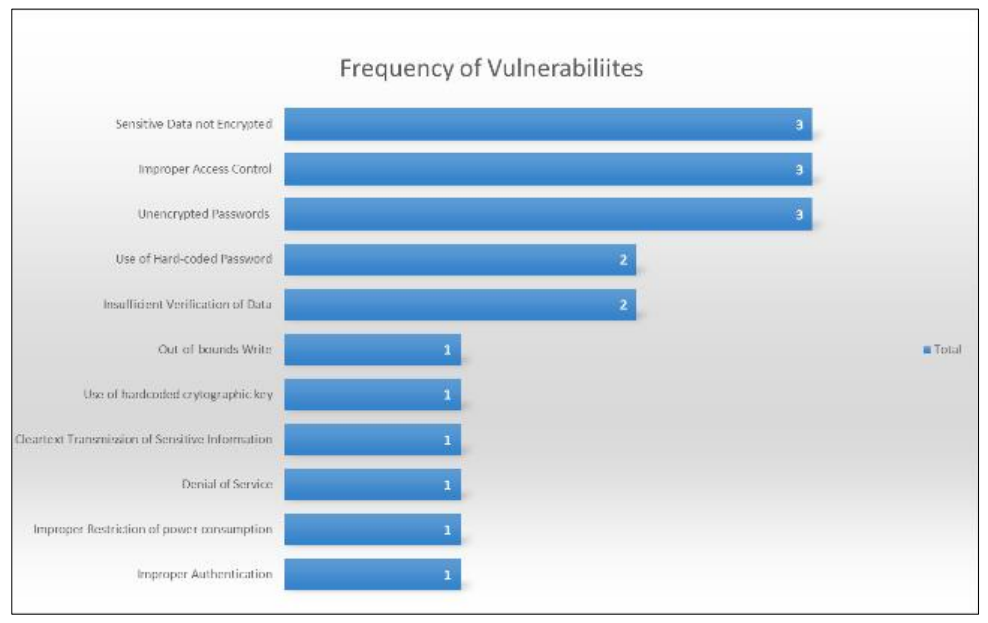

Figure 1. Published vulnerabilities on Abbott, Boston Scientific and Medtronic medical devices

For the most part, it was determined that the vendors' mitigation techniques included either over-the-air product updates or patches installed during regular office visits. Medtronic also has an active commitment to Cyber security and are on the record stating "Medtronic firmly believes that the therapeutic benefits of our products far outweigh any potential security risks". They continuously monitor the security of their products and take immediate actions to remediate. This can be said for the other leading medical device manufacturers researched in this paper. One of the main reasons for this is the US Food and Drug Administration (FDA). The FDA has implemented guidelines or steps in the mitigation of cybersecurity risks. Both medical device manufacturers (MDMs) and Healthcare delivery organizations are responsible for remaining vigilant when identifying cybersecurity risks associated with their medical devices and the networks these devices are connected to. Both must work together to ensure appropriate mitigations are in place.

From the results obtained in this research there has been no known active exploit of any of the vulnerabilities identified. However with the rise of cyber-attacks over the past number of years, especially within the healthcare sector, the possibility of exploit comes even closer.

The initial research approach hoped to find extensive vulnerability information and management practices available for older medical IoT devices, of which many exist. However, there was once again too little information available to produce a viable data set in order to complete part B of the research question, "what measures should be in place to mitigate or prevent these vulnerabilities on older IoT devices". With this shortcoming, instead a Vulnerability Risk Assessment Tool (VRAT) is proposed which will allow a vendor to determine the overall cybersecurity risk an IoT device could pose. VRAT will take security considerations as inputs, such as the age of the device as well as other parameters like security controls and mitigation techniques. It will then output an advisory or recommended action according to the risk rating.

The approach is based off the NIST Risk Management Framework. This framework outlines a process which integrates security and risk management into the system development life cycle (SDLC) and considers effectiveness, efficiency and constraints according to industry regulations (Medtronic, n.d.). The framework consists of six steps: Categorize system, Select controls, Implement controls, Assess controls, Authorize system and Monitor controls.

The framework is mapped to the tool, with additional categories per step added. A risk rating is then produced which will determine a vulnerability management course of action, thus fulfilling the second part of the research question.

Using the data obtained in the medical device research as inputs for the added categories, VRAT was created by assigning a risk value to the following:

a) Added Category: Vulnerability types according to severity. 
b) The impact a particular device has according to the CIA triad.

c) The security controls required and implemented for the device.

d) Added Category: The categories of device age.

e) The levels of information an organisation has about its devices.

f) Added Category: Any mitigation techniques the vendor has in place.

A calculation was then created to determine the overall risk score achieved out of 10 . Based on this rating, an advisory was outputted.

The Categorize step of the framework is used to determine an impact level - low, medium or high. These relate to the level of importance a device has. Each level is assigned a score as per Table 1. A categorization of vulnerability type is added also and assigned a risk score of between 1 and 10 based on the severity of the vulnerability type should it be exploited.

The values given to the vulnerability type in these examples are based on the dataset provided. In general, the values are discretionary and are based on different factors considering the situation. For example, in the medical device industry, management of PII is imperative, therefore a vulnerability type which allows sensitive information to be transmitted without encryption is a high risk and has been allocated a score with ten, rated the most severe. If however, the device was a mundane object like a fridge, the score would be much lower as the risk of exploiting sensitive information would be minor. Additionally, if there were multiple vulnerability types added, some of which could be a higher severity than cleartext transmission of sensitive information, these vulnerability types could be assigned a higher value and cleartext transmission of sensitive data could drop to a nine. Similarly, the impact scores are weighted accordingly with high impact awarded the most as it applies to the most critical asset. The overall Categorize value will be the addition of both fields divided by 2 .

Table 1. Categorize Step Values

\begin{tabular}{|l|l|l|l|l|}
\hline \multicolumn{5}{|c|}{ Datasets } \\
\hline \multicolumn{1}{|c|}{ Vulnerability Type } & Value & & Impact Level & Value \\
\hline $\begin{array}{l}\text { Cleartext Transmission of } \\
\text { Sensitive Information }\end{array}$ & 10 & & High & 10 \\
\hline Denial of Service & 9 & & Low & 1 \\
\hline Improper Access Control & 6 & & Medium & 5 \\
\hline $\begin{array}{l}\text { Improper Restriction of } \\
\text { power consumption }\end{array}$ & 4 & & & \\
\hline $\begin{array}{l}\text { Insufficient Verification of } \\
\text { Data }\end{array}$ & 7 & & & \\
\hline Out of Bounds Write & 5 & & & \\
\hline Sensitive Data not Encrypted & 10 & & & \\
\hline Unencrypted Passwords & 8 & & & \\
\hline $\begin{array}{l}\text { Use of hardcoded } \\
\text { cryptographic key }\end{array}$ & 7 & & & \\
\hline Use of Hard-coded Password & 7 & & & \\
\hline
\end{tabular}

The Select step determines the controls required for the device and any associated data, for example with medical devices some security controls would be FDA regulated. The measures used here will be: adequate, needs improvement or information not available, with values as per Table 2. "Device age" categories are additionally added to VRAT and assigned a risk score of between 1 and 10 as determined by the age of the device. The Select value will be the addition of both fields divided by 2 . 
The values assigned for the device age scales from low for the youngest device to high for the oldest. This is because the older the device the more vulnerable it is likely to be and therefore the highest risk. Having the correct security controls in place, especially for example with a medical device, is very important when it comes to keeping it secure. To allow for this, when information on the controls isn't available, implying they aren't in place or adequately monitored, this is assigned the highest value of ten, whereas an adequate rating is assigned a one for low risk.

Table 2. Select Step Values

\begin{tabular}{|l|l|l|l|l|}
\hline \multicolumn{5}{|c|}{ Datasets } \\
\hline \multicolumn{1}{|c|}{ Security Control } & \multicolumn{1}{|c|}{ Value } & & Age of Device & Value \\
\hline Adequate & 1 & & $<1$ & 1 \\
\hline Information not Available & 10 & & $<3$ & 3 \\
\hline Needs Improvement & 5 & & $<5$ & 5 \\
\hline & & & $>10$ & 10 \\
\hline & & & $>5$ & 8 \\
\hline
\end{tabular}

The Prepare step is any essential activities carried out to help an organization maintain a good security posture, including complete knowledge of asset criticality and architecture, up to date risk assessments and system information. Similar to the Select step, the measures used here will be: adequate, needs improvement or information not available, with values as per Table 3. A measure of preparedness with regard to patching or any other mitigating factors is also added and assigned a risk score of between 1 and 10. The Prepare value will be the addition of both fields divided by 2 .

The values assigned in the prepare step can be used with any device. The mitigation techniques are rating according to the effectiveness of the practice. A score of ten is given when there is no mitigation technique in place as this would pose the highest risk, however, when a device is decommissioned, this is given a three as it is adequate mitigation. The three value is given instead of a 1 as it the device could continue to pose a risk until the decommission process is complete. The values for information are the same as those given in the Select step.

Table 3. Prepare Step Values

\begin{tabular}{|l|l|l|l|l|}
\hline \multicolumn{5}{|c|}{ Datasets } \\
\hline \multicolumn{1}{|c|}{ Information } & Value & & \multicolumn{1}{c|}{ Mitigation } & Value \\
\hline Adequate & 1 & & Consistent Patching & 1 \\
\hline Information not Available & 10 & & Vulnerability Scanning & 5 \\
\hline Needs Improvement & 5 & & No mitigation Technique & 10 \\
\hline & & & Replacement of Device & 3 \\
\hline & & & Decommission of Device & 3 \\
\hline
\end{tabular}

\section{EXPERIMENTAL IMPLEMENTATION}

As each value as presented in the tables above change, so does the risk rating and the advisory given. The flow of VRAT is illustrated in Figure 2 below. 


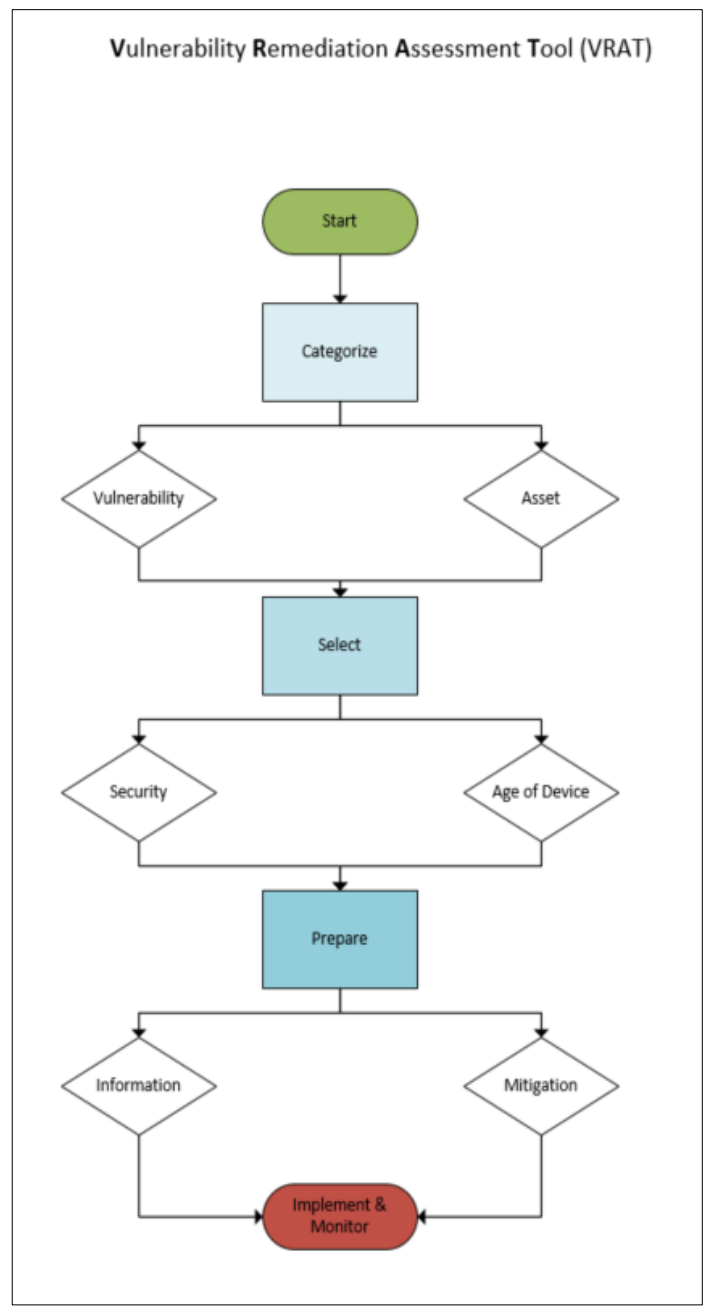

Figure 2. VRAT score calculation

The calculation to obtain the risk rating is as follows:

((Categorise: Vulnerability

$$
+ \text { Categorise: Asset)/2 }
$$

+ (Select: Security Controls

$$
\text { + Select: Age of Device)/2 }
$$

+ (Prepare: Information

$$
\text { + Prepare: Mitigation)/2))/3 }
$$

A Risk Rating of 10 is critical, whereas 1 is very low risk.

The recommended vulnerability management practice outputted is pertinent to the risk rating value as shown below in Table 4. The advisories are provided as example only for the purposes of illustration of the tool. They should be relevant to each organizations specific security program and Key Performance Indicators. 
Table 4. Output from risk rating

\begin{tabular}{|c|c|}
\hline \multicolumn{2}{|c|}{ Datasets } \\
\hline Advisory & Risk Rating \\
\hline Security Posture is adequate- maintain & 1 \\
\hline Security Posture is adequate - maintain & 2 \\
\hline Risk is low, monitor and maintain & 3 \\
\hline Risk is low, monitor and maintain & 4 \\
\hline Medium Risk, ensure up-to-date patch management & 5 \\
\hline Medium Risk, ensure up-to-date patch management & 6 \\
\hline High Risk, security program review needed & 7 \\
\hline High Risk, security program review needed & 8 \\
\hline Critical - Immediate action required & 9 \\
\hline Critical - Immediate action required & 10 \\
\hline
\end{tabular}

Figure 3 provides a sample output from VRAT in action. In this scenario, the risk rating is 8 and the parameters that led to this result are as follows:

Categorise Step:

- The vulnerability type is "cleartext transmission of sensitive information", rated 10. This vulnerability is of particular high risk to the medical device industry due to the nature of the data involved.

- The asset in question is "high", rated 10. This means this asset is of critical importance.

- The overall risk rating for this step is 10 .

Select Step:

- The security controls have "information not available", rated 10. This means the organization does not have adequate information about what controls are in place, implying perhaps that there are none. This can be a breach of regulations within the medical device industry.

- The age of the device is greater than 10 years old, rated 10. The older the device, the higher the security risk it poses.

- $\quad$ The overall risk rating for this step is 10 .

Prepare Step:

- The information available about the device has a "needs improvement" rating, with a value of 5. This means that security information on the device is available but is not up to standard.

- The mitigation for this device is "decommission of device", rated 3. The device is going to be decommissioned which means that it will no longer be on the network or pose a risk. However, as this can be a lengthy process, there is still a value of 3 assigned and when the other inputs are taken into consideration, the overall risk for the device remains high.

- $\quad$ The risk rating for this step is 4 .

The advisory for the overall risk rating of 8 is "High Risk, security program review required". Here the recommended action is for a review of the security measures in place for this device, and additionally the program as a whole. This is to ensure adequate vulnerability management practices are in place for all devices, not just this one in question. 


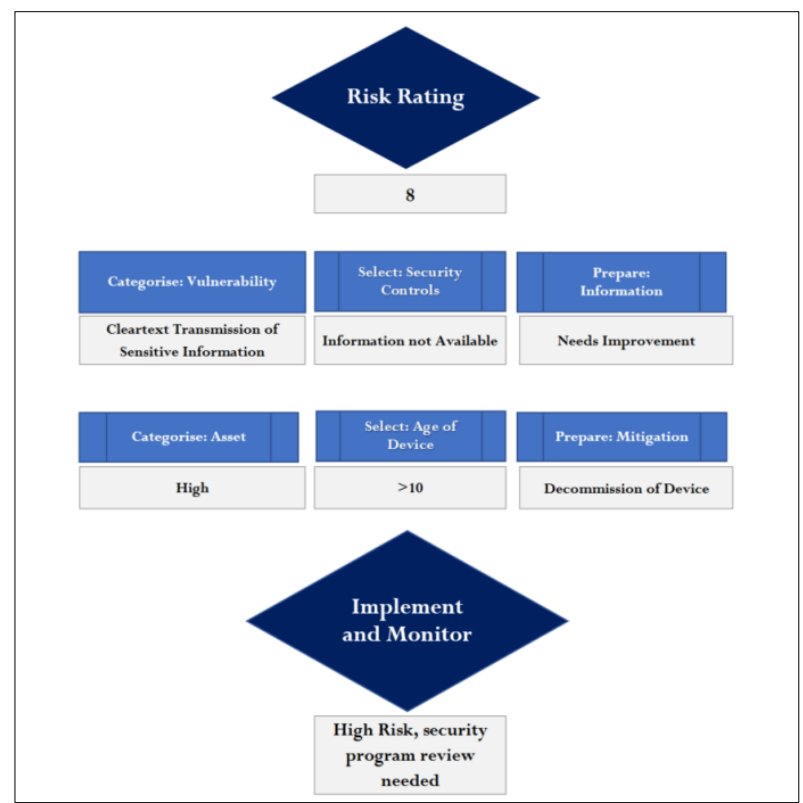

Figure 3. Parameters and advisory output for a Risk Rating of 8

\section{FUTURE WORK}

VRAT has been utilized in the examples above using data obtained from the medical device industry, however, the value of the tool lies in the fact that it is applicable to any IoT device. Used as a whole it is very comprehensive and takes multiple inputs that are pertinent to a device and/or an organization, however, it can be configured to output a rating based on any one or a number of the set parameters. The data used to drive the risk rating can be adapted and changed as required, as can the suggested advisory.

The individual inputs and values used in this paper are for illustrative purposes only to show the workings of VRAT, however, for future work a thorough list of vulnerability types should be added, as well as additional mitigation measures as relevant. Device type could be added as an input in lieu of another parameter, such as prepare: information, which uses similar data. Consideration here would be given to vulnerability types and how they could affect additional devices on the network, for example with a wormable malware and remote code execution. The advisory in such an instance would be critical.

With an increase of data in the back end, it would be more accurate to base the scoring out of 100 instead of 10 . The calculation could be modified as deemed necessary to fit requirements with consideration given to inputs that have a value of 0 which could skew the data. Further, with frequent use of VRAT, the data inputs and outputs could be automated to reduce resource overhead.

\section{CONCLUSION}

With the vast rise of internet connected devices over the past number of years comes a vast increase in the scale and variation of cyber-attacks on these devices. This paper set out to review what kind of vulnerabilities IoT devices of all ages are susceptible to and what state of the art vulnerability management techniques are available to address those threats, and we have established through the many cyber weaknesses and incidents evidenced that a significant amount of threat to data, connectivity, and increasingly health and wellbeing exists. Additionally, it is clear that the majority of research and guidelines are focused on the new and future state, rather than legacy devices which pose an even higher risk. 
As many cyber incidents are without precedence, it is becoming ever incumbent on vendors to ensure IoT devices are fully security hardened and protected against nefarious activity. The thought leadership on vulnerability management is vast, however surprisingly, our research has shown that with the growth in IoT devices comes an almost decline in adhering to these security considerations and putting capabilities in place to defend against the unknown attack, despite the many state of the art practices available. The research concluded by the SOHOpelessly Broken project (Level 3 Threat Research Labs, 2016) is perhaps the most telling given the fact that their attacks took place on fully updated devices, illustrating that the issues found lay in poor security configuration, not specifically because of lack of update as one would assume.

It remains evident that, despite the enormous benefits the IoT industry brings to consumers, enormous care must additionally be taken to ensure these devices are secure and protected against potential cyber-attack. Otherwise, the cost of convenience could come at a significant price.

\section{REFERENCES}

Angrishi, K. (2017). Turning Internet of Things (IoT) into Internet of Vulnerabilities (IoV): IoT Botnets (pp. 1-17).

Beardsley, T. (2016). R7-2016-07: Multiple Vulnerabilities in Animas OneTouch Ping Insulin Pump. Rapid7 Blog. Retrieved from https://blog.rapid7.com/2016/10/04/r7-2016-07-multiple-vulnerabilities-in-animas-onetouch-pinginsulin-pump/.

Beavers, J., Faulks, M., \& Marchang, J. (2019). Hacking NHS Pacemakers: A Feasibility Study. Proc. 12Th Int. Conf. Glob. Secur. Saf. Sustain., ICGS3 2019, 206-212.

Classen, J., Wegemer, D., Patras, P., Spink, T., \& Hollick, M. (2018). Anatomy of a Vulnerable Fitness Tracking System: Dissecting the Fitbit Cloud, App, and Firmware (pp. 1-24). ACM Interactive, Mobile, Wearable Ubiquitous Technol, vol 2.

Cyr, B., Horn, W., Miao, D., \& Specter, M. (2014). Security Analysis of Wearable Fitness Devices (Fitbit) (pp. 1-14). Massachusetts Inst. Technol.

Evans, D. (2011). IoT by Cisco 2011.pdf. Retrieved from http://census2012.sourceforge.net/paper.html.

Harwood, T. (2019). Internet of Things (IoT) History. Retrieved 6 March 2020, from https://www.postscapes.com/iothistory/.

IDC. (2020). The Growth in Connected IoT Devices Is Expected to Generate 79.4ZB of Data in 2025, According to a New IDC Forecast. IDC: The premier global market intelligence company. Retrieved from https://www.idc.com/getdoc.jsp?containerId=prUS45213219.

Kirk, J. (2012). Pacemaker hack can deliver deadly 830-volt jolt. Computerworld. Retrieved from https://www.computerworld.com/article/2492453/pacemaker-hack-can-deliver-deadly-830-volt-jolt.html.

Level 3 Threat Research Labs. (2016). Attack of Things!. Level3. Retrieved from http://blog.level3.com/security/attackof-things/.

Lofty, K., \& Hale, M. (2016). Assessing pairing and data exchange mechanism security in the wearable internet of things. In IEEE Int. Conf. Mob. Serv. (pp. 25-32).

McMahon, E., Williams, R., El, M., Samtani, S., Patton, M., \& Chen, H. (2017). Assessing medical device vulnerabilities on the Internet of Things. IEEE Int. Conf. Intell. Secur. Informatics Secur., 176-178.

Mirani, S., Meyer, J., Rarngattie, R., \& Sindermann, I. (2019). SOHOpelessly Broken 2.0: Security Vulnerabilities in Network Accessible Services, 1-34.

Rieck, J. (2016). Attacks on Fitness Trackers Revisited: A Case-Study of Unfit Firmware Security (pp. 33-44).

Zheng, G., Zhang, G., Yang, W., Valli, C., Shankaran, R., \& Orgun, M. (2017). From WannaCry to WannaDie: Security trade-offs and design for implantable medical devices. 17Th Int. Symp. Commun. Inf. Technol. Isc., 2018-January, $1-5$.

Burhan, M., Rehman, R., Khan, B., \& Kim, B. (2018). IoT elements, layered architectures and security issues: A comprehensive survey (pp. 1-37).

Fitbit. (2019). Fitbit Legal Privacy Policy. Fitbit.com. Retrieved from https://www.fitbit.com/us/legal/privacy-policy.

Franklin, C. (2019). How to make sure your fitness trackers are secure. The Verge. Retrieved from https://www.theverge.com/2019/8/28/20825680/fitness-trackers-privacy-security-safe-how-to-fitbit-apple-watchgarmin.

Hyppönen, K., \& Haataja, K. (2007). Niño’ man-in-the-middle attack on bluetooth secure simple pairing. In 2007 3rd IEEE/IFIP Int. Conf. Cent. Asia Internet (pp. 1-5). 
ICS-CERT Advisories. (2016). ICS Advisory (ICSMA-16-279-01) Animas OneTouch Ping Insulin Pump Vulnerabilities. Retrieved from https://www.us-cert.gov/ics/advisories/ICSMA-16-279-01.

Kantaria, P. (2018). Rise of the IoT botnet: Bot activity in the Internet of Things has doubled. Verdict. Retrieved from https://www.verdict.co.uk/rise-of-the-iot-botnet/.

Kumar, N., Madhuri, J., \& Channegowda, M. (2017). Review on security and privacy concerns in internet of things. In IEEE Int. Conf. IoT its Appl..

Marks, T. (2019). The Privacy Risks of Your Fitness Tracker. VPNoverview.com. Retrieved from https://vpnoverview.com/privacy/devices/privacy-risks-fitness-tracker/.

Nagy, L., \& Colesa, A. (2019). Router-based IoT Security using Raspberry Pi. In RoEduNet IEEE Int. Conf. (pp. 1-6).

Nield, D. (2019). How to Lock Down Your Health and Fitness Data. Wired. Retrieved from https://www.wired.com/story/health-fitness-data-privacy/.

NIST. FISMA Implementation Project. Csrc.nist.gov. Retrieved from https://csrc.nist.gov/Projects/risk-management/rmfoverview.

Sizemore, A., \& Craig, S. (2016). Are Fitness Bands Secure? There's More to It Than Just the Clasp!. Security Intelligence. Retrieved from https://securityintelligence.com/are-fitness-bands-secure-theres-more-to-it-than-just-theclasp/.

U.S Food and Drug Administration. (2020). Cybersecurity. U.S. Food and Drug Administration. Retrieved from https://www.fda.gov/medical-devices/digital-health/cybersecurity.

Vlajic, N., \& Zhou, D. (2018). IoT as a Land of Opportunity for DDoS Hackers (pp. 26-34).

Wan, D. (2019). Targeting Cybersecurity Investment - a FAIR Approach. Fairinstitute.org. Retrieved from https://www.fairinstitute.org/blog/targeting-cybersecurity-investment-the-fair-approach.

Medtronic. Product Security and Cybersecurity. Global.medtronic.com. Retrieved from https://global.medtronic.com/xgen/product-security.html. 